\title{
Health financing lessons from Thailand for South Africa on the path towards universal health coverage
}

Five years after the release of its Green Paper on National Health Insurance (NHI), ${ }^{[1]} 4$ years after the institution of NHI pilot sites and following the recent release of the White Paper on NHI, ${ }^{[2]}$ South Africa (SA) needs to move beyond the phase 1 plans , policy making and health system strengthening activities to phase 2 - putting into place the legal and institutional frameworks and systems for implementation of its universal health coverage (UHC) system. In doing so, SA can draw on considerable practical lessons from other countries' reforms in managing UHC with favourable equity outcomes over the past decade. ${ }^{[2,3]}$ We outline some potentially significant lessons from the Thai health financing system for SA.

Thailand has received widespread international recognition as one of several middle-income countries that have made enormous progress in building a UHC system and in achieving 'good health at low cost: ${ }^{[4]}$ Although its per capita GDP is below that of SA, Thailand has not only massively improved health outcomes (e.g. infant mortality $9.8 / 1000^{[4]}$ ) but made great improvements in social security objectives ( $>99 \%$ population coverage, high level of financial risk and impoverishment protection). It has low out-of-pocket payments and health-related catastrophic expenditure has fallen from $2.7 \%$ to $0.49 \%,{ }^{[5]}$ but there is some room for improvement for urban poor populations.

SA can learn a range of practical lessons from Thailand's experience. Firstly, SA wants to establish an NHI fund. Thailand has a strong national fund called the Universal Coverage (UC) Fund, established in 2002 , which covers $75 \%$ of its population, the rest being covered by social health insurance and the Civil Servant Medical Benefit Scheme (CSMBS). Thailand has a well-developed purchaser-provider split, with the independent UC Fund established by legislation, with a multi-stakeholder governing body including private and civil society representatives. Its internal structure, operating systems, procedures and information technology are firmly established, accessible and affordable in the middle-income country context. In the early stages, both countries' funds will cover the majority of the population not covered by other insurance arrangements: medical schemes in the case of SA ( $16 \%$ of the population), and social security (15\%) and the CSMBS (9\%) in the case of Thailand. Both funds will initially focus mainly on public sector provision, but will progressively explore mixed public and private provision options (for example, Thailand has used private healthcare providers to clear a large cataract backlog). Despite fairly sophisticated population registration, reimbursement and audit systems, the administration cost of the Thai UC Fund is a remarkably efficient $1.2 \%$ of its total annual budget. The National Health Security Office (NHSO), which manages the UC Fund, concentrates on pooling and strategic purchasing; it has no revenue collection function, as the scheme is financed through an annual budget. The successes of the UC Fund are such that the CSMBS and social security have adopted a number of its systems (e.g. diagnosis-related groups (DRGs)). In addition, the NHSO manages the disease prevention and health promotion budget for all Thai citizens, thus assisting the other schemes and providing a strong focus on prevention and promotion beyond the conventional scope of insurance.

Secondly, SA is keen to embark on reimbursement reform and put in place a system of capitation for primary care and DRGs for hospital inpatients in order to contain hospital spending, adjust for case-mix severity, fund primary care more equitably and improve technical efficiency. ${ }^{[6]}$ While the development of such systems is still in early planning phase in SA, they have been practically implemented and refined in Thailand over several years. Thailand's civic registration system (covering $98.4 \%$ of total births and deaths ${ }^{[7]}$ ) operates mainly through the national ID card and is an impressive sign of citizenship; all citizens are visible, along with their health provider unit in the electronic system of the UC Fund, at every hospital and primary care centre. The Thai capitation system uses an interesting contracting unit called the CUP (Contracting Unit for Primary Care), which is the district hospital along with its referring primary care centres. The UC Fund contracts with 800 CUPs, each with a manageable number of 50000 catchment population, ${ }^{[5]}$ which is operationally feasible, while within each CUP the district hospital and its primary care centres work together, sharing the outpatient capitation budget from the UC Fund and collaborating as a horizontally integrated provision unit. The Thai DRG system, now in its version 5.1, is well developed and has drawn on the Australian grouper systems in its development. Both hospitals and health centres in Thailand have their own bank accounts and can retain surplus revenue for operation and additional incentives for staff. This decentralised holding of funds has provided greater flexibility to health facilities to solve local problems. These use simpler accounting systems and are audited by the auditorgeneral. In addition to the main capitation and DRG reimbursement mechanisms, certain expensive and prioritised activities also receive additional payments to encourage their provision.

Thirdly, Thailand has a very deep primary care system with over 10000 public health centres ${ }^{[8]}$ (approximately $1 / 6000$ population $\mathrm{v}$. $1 / 13000$ in SA). In addition to nursing and public health officers, each health centre has up to 30 community health volunteers who also work closely with health centres and local government on prevention and promotion. Substantial investments were made in infrastructure for rural health centres, often using attractive but standardised design options, lowering infrastructure costs. In a farsighted funding arrangement, every CUP (and therefore district hospital and health centres) receives a dedicated funding stream for prevention and promotion activities from the UC Fund. In addition, each CUP has a co-funding arrangement with the local government for prevention, promotion, rehabilitation and public health, where the local government contributes between $10 \%$ and $50 \%$ depending on fiscal capacity. ${ }^{[9]}$ Thailand also has a national Health Promotion Foundation, established by law as an independent public organisation and funded through a mandatory $2 \%$ additional surcharge on excise tax on alcohol and tobacco, which implements a wide variety of nonclinical health promotion activities using a wide range of media and stakeholders.

Fourthly, Thailand has achieved impressive health outcomes, financial risk protection, and universal coverage with advanced reimbursement systems at remarkably low cost. With the progression towards UHC, public health expenditure has grown from only $3.4 \%$ of the GDP in 2005 to $5.6 \%$ in 2014 , and total health expenditure from $4.6 \%$ to $6.5 \% .^{[10]}$ Many factors contribute to its low costs, particularly on the supply side. Total inpatient payments using DRGs are capped, primary care spending is limited by the capitation mechanisms, and there is substantial use of central procurement of certain high-cost medicines by the NHSO and use of generic 
medicines by contractor providers. The primary care system is highly accessible; patients who bypass their registered CUP are liable to pay full user fees. CUP fund holding is applied, by which higher-level hospitals can bill lower-level hospitals for OPD services (money follows the patient). The use of one main large UC Fund simplifies administration. Relatively low wage costs along with a strong work ethic and the use of mainly public providers contribute to the low costs. There is a sophisticated process of and institutional capacity for health technology assessment using economic evaluation techniques before new expensive interventions and medicines are included into the benefit package of the UC Fund.

The Thai Ministry of Public Health and the UC Fund are supported by a number of independent or semi-independent institutions that provide independent technical support. These include, among others, the International Health Policy Program and its capacity development arm for UHC, the Health Intervention and Technology Assessment Program (HITAP), which undertakes economic evaluation of new interventions, the Centre for Health Equity Monitoring and the Thai CaseMix Centre, which develop and refine the DRG system, the Thai Health Foundation and the Healthcare Accreditation Institute. SA could benefit from a health technology assessment institution like the HITAP to assess new interventions and technologies and refine the NHI benefit package over time.

However, Thailand and SA also differ in important ways that may make their health financing systems differ. One of the most important of these is that provinces in SA have greater legally derived powers than in Thailand. The relative role and co-ordination between the centre and the provinces still needs to be finally resolved in the SA reforms and in the legislation that defines SA's new UHC system. While SA has a large private medical scheme system, Thailand has significant CSMBS and social security funds outside the UC Fund. For both countries the progressive alignment and ultimate integration of funding pools into a single fund, as the universal arrangements are progressively built and strengthened, will remain a key agenda. Both countries face a challenge to find the right mix of public and private provision. Although the Thai UC system uses mainly public provision (public sector infrastructure, services and benefits were strengthened over several decades, and the number of health science graduates was increased), public primary care in urban cities remains weak while the private sector concentrates on tertiary specialised care. Thailand has a strong market economy that has achieved large GDP improvements over two decades, rising from a low-income to an upper middle-income country. It has a relative small private sector ( $25 \%$ and $21 \%$ of hospitals and beds, respectively, and spending considerably less than in SA), and both countries will be challenged to find a more diverse provision mix as wealthier urban populations grow. The Thai UC Fund has built strong administrative capacity, reimbursement tools and audit mechanisms that will allow for contracting with a wider range of provider options.

\section{Mark Blecher}

National Treasury, South Africa

mark.blecher@treasury.gov.za

\section{Anban Pillay}

National Department of Health, South Africa

\section{Walaiporn Patcharanarumol, Warisa Panichkriangkrai,} Viroj Tangcharoensathien

International Health Policy Program, Ministry of Public Health, Thailand

\section{Yot Teerawattananon}

Health Intervention and Technology Assessment Program, Ministry of Public Health, Thailand

\section{Supasit Pannarunothai}

Centre for Health Equity Monitoring Foundation, Thailand

\section{Jonatan Davén}

National Treasury, South Africa

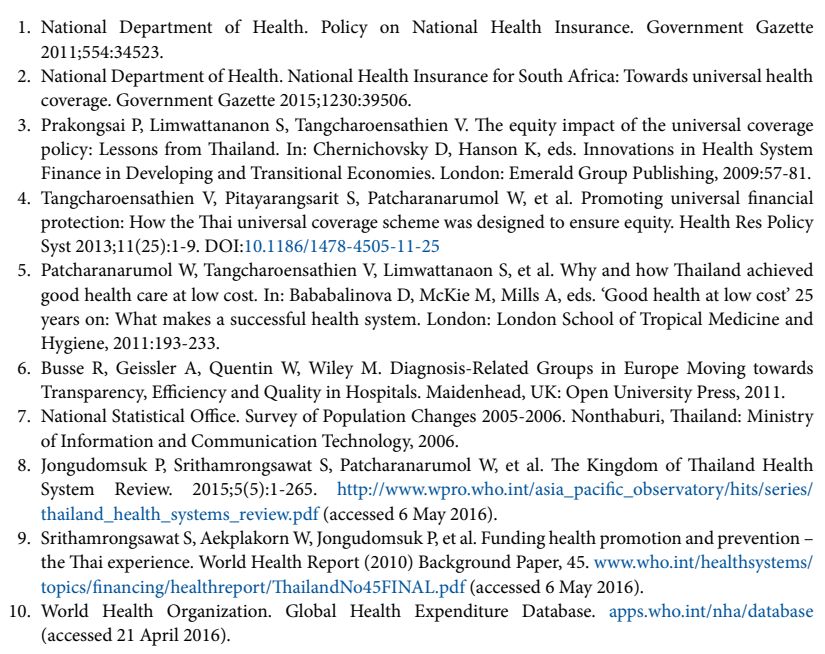

S Afr Med J 2016;106(6):533-534. DOI:10.7196/SAMJ.2016.v106i6.10953 